\title{
Análisis de accesibilidad urbana a partir de intervenciones viales mediante sistemas de información geográfica. Caso de estudio, la malla vial del municipio de Quibdó, en Colombia
}

\author{
Jorge A. Montoya(1), Diego A. Escobar ${ }^{(1) \star}$ y Carlos A. Moncada ${ }^{(2)}$ \\ (1) Universidad Nacional de Colombia - Sede Manizales. Facultad de Ingeniería y Arquitectura, Departamento de \\ Ingeniería Civil, Grupo de Investigación en Movilidad Sostenible. Carrera 37 con Calle 94, Campus La Nubia, Manizales, \\ 170003, Colombia. (correo-e: joamontoyago@unal.edu.co; daescobarga@unal.edu.co) \\ (2) Universidad Nacional de Colombia - Sede Bogotá. Facultad de Ingeniería, Departamento de Ingeniería Civil y Agrícola, \\ Programa de Investigación en Tránsito y Transporte. Ciudad Universitaria edificio 214 oficina 417, Bogotá, 111321, \\ Colombia. (correo-e: camoncadaa@unal.edu.co)
}

* Autor a quien debe ser dirigida la correspondencia

Recibido Abr. 4, 2019; Aceptado May. 30, 2019; Versión final Jul. 31, 2019, Publicado Abr. 2020

\begin{abstract}
Resumen
La presente investigación busca establecer el nivel de impacto generado por la implementación e intervención de la malla vial del municipio de Quibdó (Departamento del Choco en Colombia), en el marco del proyecto Plan Integral de Movilidad para un periodo de corto plazo (2025). Dicho plan fue realizado por la Universidad Nacional de Colombia, Sede Manizales y la administración municipal en el año 2015. La metodología aborda el concepto de accesibilidad territorial urbana como modelo de evaluación del impacto que generarán las intervenciones viales propuestas por el Plan en el corto plazo una vez construidas. Se usan herramientas digitales (ArcMap, Microsoft Excel, TransCad) y se vinculan variables socioeconómicas (área, población, viviendas) en el análisis. Como principal resultado, se obtiene que las intervenciones viales propuestas en el plan, generan un menor tiempo de acceso hacia los sectores norte, sur y occidental del municipio; con lo cual se puede concluir que las intervenciones propuestas se encuentran adecuadamente direccionadas desde el punto de vista de disminución de tiempos medios de viaje.
\end{abstract}

\section{Urban accessibility analysis from road interventions through geographic information systems. Case Study, the road network of the Quibdó municipality, in Colombia}

\begin{abstract}
This research seeks to establish the level of impact generated by the implementation and intervention of the road network of the municipality of Quibdó (Department of Choco in Colombia), within the framework of the project Integral Mobility Plan for a short term (2025). This plan was carried out by the National University of Colombia, Manizales branch, and the municipal administration in 2015. The methodology addresses the concept of urban territorial accessibility as a model for evaluating the impact generated by road interventions proposed by the Plan in the short term, once they are built. Digital tools are used (ArcMap, Microsoft Excel, TransCad) and socio-economic variables (area, population, housing) are introduced into the analysis. As a main result, it is found that the road interventions proposed in the plan, generate a shorter access time to the northern, southern and western sectors of the municipality. This leads to conclude that the proposed interventions are adequately addressed from the point of view of decreasing average travel times.
\end{abstract}

Keywords: accessibility; infrastructure; coverage; travel time; saving gradient 


\section{INTRODUCCIÓN}

Geográficamente, el espacio urbano está conformado por: i) el espacio de producción, ii) el espacio social, y iii) el espacio de circulación (Blanco, 2016), concepto que se pone aprueba en la presente investigación al establecer la relación entre la operatividad del llamado espacio de circulación y los otros dos tipos de espacios según una variable cuantificable como lo es el tiempo medio de viaje, en donde el concepto de red se configura como la nueva forma de comprender el espacio urbano (Dupuy, 1998). Quibdó, capital del Departamento del Chocó, se identifica como ciudad funcional, al corroborarse como un área de tratamiento administrativo y de gestión en el cual se desarrollan actividades típicamente no primarias (Kralich, 2016), resaltándose la jerarquía lograda por la ciudad central y la dependencia de su entorno, lo cual se encuentra íntimamente ligado al conjunto de intervenciones viales propuestas en el Plan Integral de Movilidad al horizonte 2025. Quibdó se localiza a $53 \mathrm{msnm}$ en la región noroccidente de Colombia sobre los $76^{\circ} 39^{\prime} 40^{\prime \prime}$ de longitud este y $5^{\circ} 41^{\prime} 13$ " de latitud norte (Figura 1); cuenta con una población de 115.711 habitantes, una superficie total del $3.337,5 \mathrm{~km}^{2}$, de los cuales menos del $1 \%$ es área urbana $\left(19,85 \mathrm{~km}^{2}\right)$ y una topografía mayormente plana. Ahora bien, en el marco del Plan Integral de Movilidad de Quibdó, el cual fue redactado, entregado a la Administración Municipal y aprobado por el Concejo Municipal en el año 2015, se estableció que el modo de transporte más usado por la población es la motocicleta, con valores superiores al $80 \%$ del volumen vehicular registrado durante la hora pico (Alcaldía de Quibdó, 2015); la longitud total del espacio de circulación automotor asciende a $198 \mathrm{Km}$, de los cuales $8 \%(16 \mathrm{Km})$ son vías principales, $4 \%(8 \mathrm{Km})$ son vías secundarias y el resto es red vial local (Ver Figura 1), encontrándose importantes deficiencias en el tipo y estado de la superficie de rodadura, en donde solo el $26 \%$ se encuentra pavimentado, y alrededor del $68 \%$ está en mal estado. Por otra parte, se percibe un fuerte incremento en el tiempo medio de viaje al momento de realizar las actividades cotidianas, teniendo que el $42 \%$ de la ciudadanía argumenta que éste se incrementó respecto al año anterior, según informes de veedurías municipales.
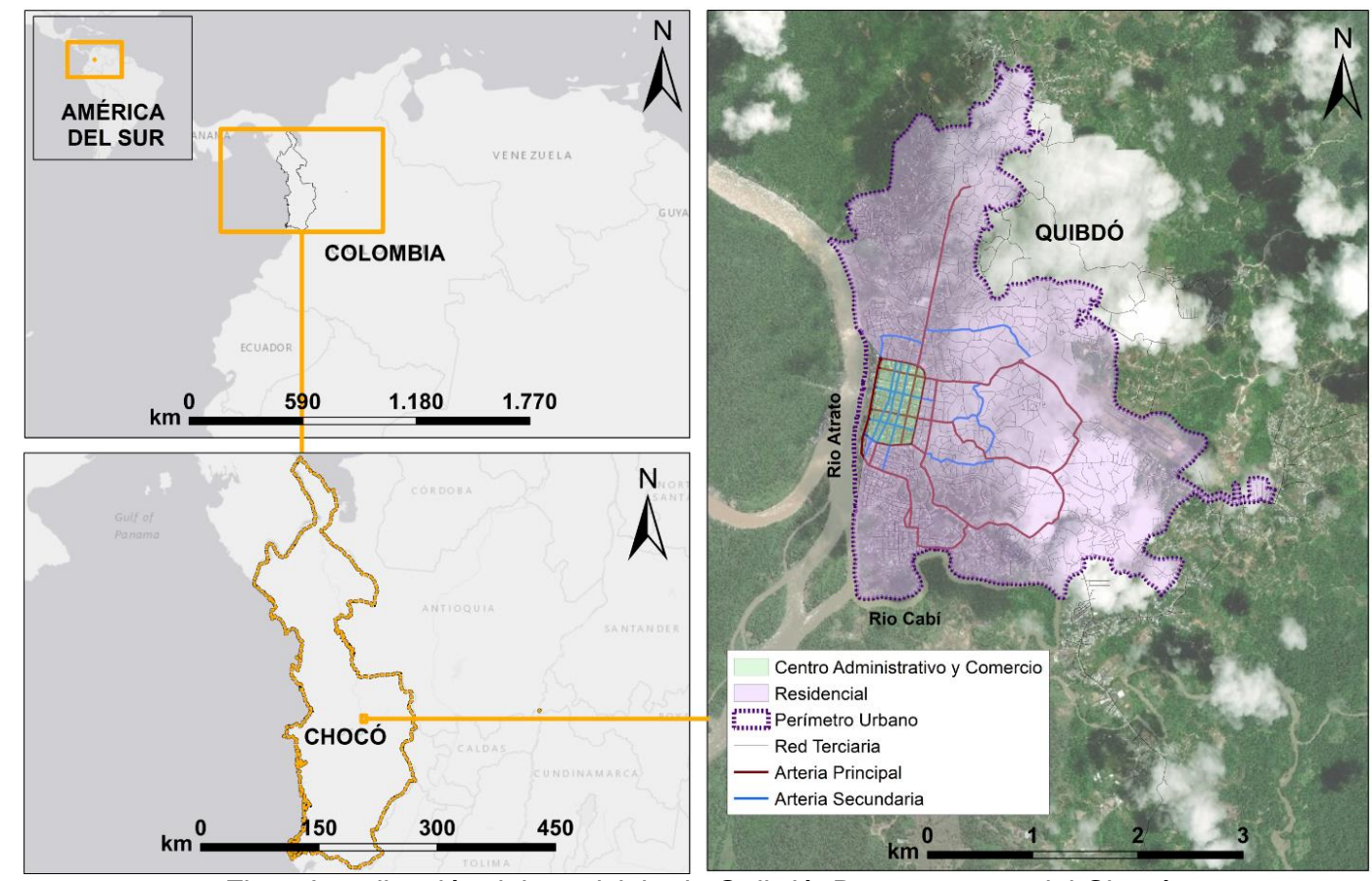

Fig.1: Localización del municipio de Quibdó, Departamento del Chocó

En Quibdó, el crecimiento del núcleo urbano original se ha dado de forma orgánica a medida que se han ido involucrando al espacio urbano los suburbios periféricos, llevando a que su denominación se relacione con la de un aglomerado (Kralich, 2016); dicho modelo de crecimiento ha aumentado la intensidad y velocidad de los flujos, lo que se traduce en un mayor uso del espacio de circulación y consecuente necesidad de control, gestión y planeamiento, al percatarse la necesidad de conectar el sector del centro administrativo y de comercio con el sector residencial que le rodea (Ver Figura 1), lo cual genera un dinámica de movilidad de tipo monocéntrica, en la que en horas de la mañana la población se desplaza hacia el sector productivo (trabajo, estudio) y en horas de la tarde regresa hacia el sector reproductivo (residencial) (Blanco, 2016). La hipótesis se basa en que al realizar procesos de mejora o rehabilitación de las infraestructuras del transporte, hay un aumento de cobertura espacial en términos de alcance permitido por las infraestructuras, lo cual a su vez, aumenta las probabilidades de elevar la calidad de vida de los habitantes, al disminuir el costo de viaje de los usuarios, lo que se relaciona directamente con las características de movilidad. Es necesario diferenciar los conceptos de movilidad y transporte, los cuales, a pesar de compartir una misma unidad de estudio (viajes), no comparten el mismo universo de los mismos (Gutiérrez, 2012). Las infraestructuras de transporte, 
permiten la interacción ciudadana con los componentes social y económico circundantes, jugando un papel clave en el desarrollo de una región dado el impacto directo e indirecto sobre la productividad (Brida et al., 2014), entendiéndose que las variadas actividades cotidianas en un territorio se desarrollan a través de soportes infraestructurales con condiciones morfológicas y de localización particulares, que dependen no sólo del motivo de realización de un viaje sino del medio de transporte utilizado y su relación espacial (Blanco, 2016). Es por ello que el transporte es necesario para conectar dos o más puntos específicos del espacio urbano o del territorio, no obstante, es insuficiente al momento de establecer la movilidad, pues el transporte no aborda la mediación existente entre sociedad y territorio; es así que el concepto "movilidad" amplía la frontera de un estudio de transporte (Gutiérrez, 2012).

Por otra parte, en esta investigación se aborda el concepto de "accesibilidad", el cual ha sido usado como instrumento de planificación urbana desde la segunda década del siglo XX (Batty, 2009), sin embargo, en la literatura se ha asumido como definición clásica la expresada por Hansen (1959), quien la define como el potencial de oportunidades para la interacción que poseen las personas en relación con las diferentes zonas que componen una aglomeración o espacio urbano. A pesar que el concepto cuente con gran número de definiciones que difícilmente pueden llegar a un consenso (Murray y Wu, 2003), es allí donde a pesar de existir un vínculo teórico entre movilidad y accesibilidad, esta última es medible de una forma relativamente sencilla, mientras que la accesibilidad ofrecida por la red de infraestructuras de transporte requiere ir más allá de conocer cuánto se mueven las personas (Hansz et al., 2018); es decir, la movilidad observada puede considerarse un componente de la accesibilidad. A través de los años la accesibilidad se ha abordado desde variados enfoques (Geurs y Van Wee, 2004; Van Wee, 2016) y medidas (Pirie, 1979). Los enfoques pueden ser considerados de forma simultánea, éstos son: i) usos del suelo, mediante el cual se analiza la cantidad, calidad, distribución espacial y demanda de las oportunidades; ii) sistemas de transporte, mediante el cual se analizan las condiciones operativas de éstos; iii) componente temporal, mediante el cual se analizan restricciones de las oportunidades según un horario o alguna característica temporal; iv) Aspectos individuales, mediante el cual se analizan necesidades o características particulares de los individuos; y v) componente cognitivo (Lucas, 2012) el cual incluye la habilidad de las personas para interactuar con los sistemas de transporte disponibles. Entre las medidas de accesibilidad se tienen tres tipos: i) relativa, la cual se calcula entre dos puntos A y B particulares de la red de infraestructuras (Pirie, 1979); ii) integral, se calcula desde o hacia uno o varios puntos específicos que representan las oportunidades como educación, trabajo, salud, etc. (Handy y Niemeier, 1997); y iii) global, en la cual se calculan los tiempos medios de viaje entre todos los nodos que componen una red de infraestructuras, siendo útil para comparar escenarios que evalúen el impacto en la accesibilidad por la construcción o mejoramiento de infraestructura (Martínez et al., 2017). Algunas aplicaciones del concepto desde áreas específicas de investigación son: usos del suelo y transporte (Escobar et al., 2015; Murray y Wu, 2003; Yigitcanlar et al., 2010), agricultura (Arcidiacono y Porto, 2010), desarrollo sustentable (Kwok y Yeh, 2004), Comercio (Montoya et al., 2017), educación (Walsh et al., 2017), seguridad vial (Geetam y Deepty, 2012), Exclusión y equidad social (Lucas et al, 2016; Schürmann y Spiekermann, 1997), entre muchas otras áreas.

Se resalta entonces que el concepto de accesibilidad toma gran importancia en la planificación del territorio y se convierte en un importante insumo para la toma de decisiones de política pública, pues dependiendo del enfoque abordado, es posible identificar las áreas del territorio y/o grupos poblacionales que registran deficiencias en las condiciones de accesibilidad dada la operatividad de la infraestructura del transporte. Hoy en día existen infinitas posibilidades de recopilar información geográfica relacionada con la ubicación de la población y la infraestructura de transporte disponible para alcanzar actividades, lo que no sólo permite la existencia de movilidad, sino que permite un análisis de ordenamiento territorial, usos del suelo y operatividad más detallado; lo anterior se convierte en una gran oportunidad para poner en marcha adecuados procesos de planificación mediante la aplicación de metodologías que identifiquen los cambios generados en los espacios geográficos (Moreno y Jácome, 2017), dada la inserción de nuevas infraestructuras, lo que a su vez, es base para la definición de políticas públicas prioritarias por parte de una administración municipal.

Teniendo en cuenta lo anterior, se evidencia la necesidad de intervenir las infraestructuras del transporte del municipio, con el fin de mejorar las condiciones de movilidad y accesibilidad desde y hacia los sectores administrativos y de comercio y residenciales. Así mismo, al igual que los diversos modos de aplicación de la accesibilidad, es posible identificar elementos comunes dentro de éstos, la red de infraestructura, el análisis de modos de desplazamiento y los elementos generadores o atractores de viajes; razón por la cual es posible afirmar que la vinculación de la accesibilidad dentro de la presente investigación, como herramienta de valoración de las intervenciones propuestas por la administración municipal, es una excelente opción. En esta investigación se plantea la elaboración de un análisis de accesibilidad media global con enfoque en el sistema de transporte que usa el espacio de circulación automotor, buscando evaluar el impacto que sobre las condiciones de accesibilidad, ejercen las intervenciones viales propuestas por la administración municipal al año 2025 (Corto plazo) en su documento formulación del Plan Integral de Movilidad; se observa entonces que se aborda una investigación basada en el análisis de un espacio urbano y no en el análisis de individuos, en 
la que a partir del cálculo del tiempo medio de viaje en los sistemas de transporte disponibles, fue posible identificar los sectores de Quibdó que registraron mayor beneficio dada la futura construcción de dichas obras de infraestructura, ya que actualmente no se han ejecutado. Diversas investigaciones han utilizado la accesibilidad como herramienta de planeación urbana en la evaluación del impacto generado por la construcción de obras de infraestructura (Hartmanna y Yeang Yng Lingb, 2016), por ejemplo en la ciudad de Manizales se pudieron identificar las áreas de la ciudad que mayor beneficio tendrían si se construyese una infraestructura tipo túnel (Martínez et al., 2017), así como identificar cual de una variedad de intervenciones sería la más beneficiosa para la población (Escobar et al., 2018).

\section{METODOLOGÍA}

Dentro del proceso constructivo en la investigación, se desenvuelven cinco fases metodológicas de carácter consecutivo, los cuales garantizan un adecuado desarrollo de la evaluación y futuras replicaciones. La secuencia metodológica se presenta en la Figura 2 y se describe a continuación.

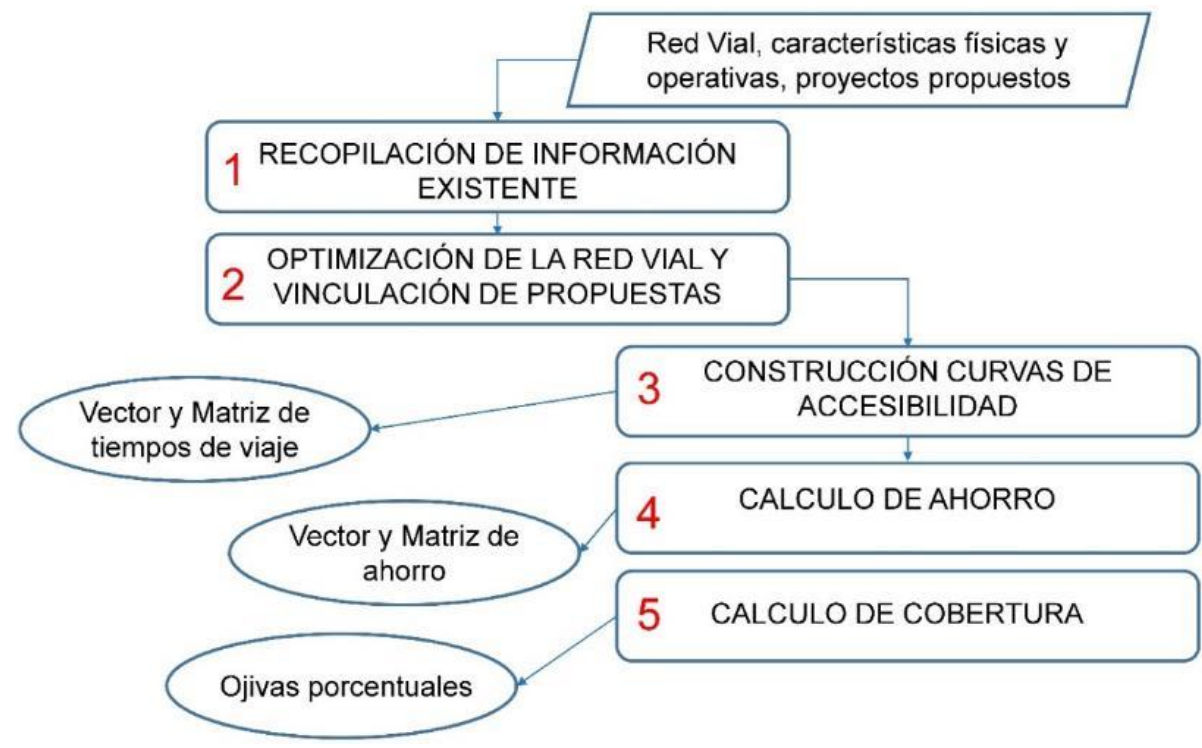

Fig. 2: Metodología de investigación.

\section{Recopilación de información existente}

Dentro de este ítem metodológico, se recopila y estructura la información existente relacionada con la red vial municipal y sus características físicas y operativas (velocidad, direccionalidad, topología, longitud, pendiente, superficie de rodadura, etc.); al igual que los proyectos propuestos por la administración municipal en el periodo corto plazo del Plan Integral de Movilidad. En relación con los datos de velocidad de operación de cada arco, éstos se obtuvieron a partir de la instalación de equipos GPS en los modos de transporte existentes en la ciudad (automóvil, motocicleta, taxi, camión y transporte público), se usaron para dicha toma de información, 25 automotores de diferentes modos, mediante los cuales se adquirieron más de 10 millones de datos de coordenadas espaciales. El grafo actual se compone por más de 10,000 arcos y 6,000 nodos. Toda esta información está consignada de forma oficial en Plan Integral de Movilidad del Municipio de Quibdó (Alcaldía de Quibdó, 2015).

\section{Validación de la red de infraestructuras del transporte y vinculación de propuestas de intervención}

En la segunda fase metodológica, se estructura la red de infraestructuras del transporte de la zona urbana, a partir del documento del Plan Integral de Movilidad y la información recopilada. Dicha red se compone de todos aquellos elementos organizativos y materiales que permiten el desplazamiento de bienes y personas, teniendo como objetivo común el superar las variables distancia - tiempo (Blanco, 2016); de forma general, una red es un conjunto de puntos de transacción (servicios públicos, ciudades, etc.) que impulsan su propia organización territorial y que evoluciona, expresando una organización del espacio y las relaciones de éste con las características esenciales de la sociedad moderna (Dupuy, 1998). Los dos elementos principales para la representación gráfica y control del funcionamiento de la red, son los Nodos (confluencia de líneas o intersecciones viales) y Arcos (elementos lineales en representación de vías o caminos) espacialmente distribuidos (Figura 3), los cuales contienen las características físicas y operativas descritas anteriormente. El proceso de vinculación de las características operativas de la red de infraestructuras se realiza mediante el software ArcMap de Esri. Una vez constituida y validada la red de infraestructuras del transporte, se procede 
a vincular los escenarios de estudio, para nuestro caso, se desarrollan dos situaciones de análisis, el escenario base, el cual establece las condiciones de accesibilidad ofertadas por la red de transportes en la condición inicial (año 2015) y el escenario futuro (año 2025); es importante recordar que el paquete de proyectos se encuentra propuesto en el documento Formulación del Plan Integral de Movilidad, considerando la construcción, rehabilitación y mantenimiento de vías.

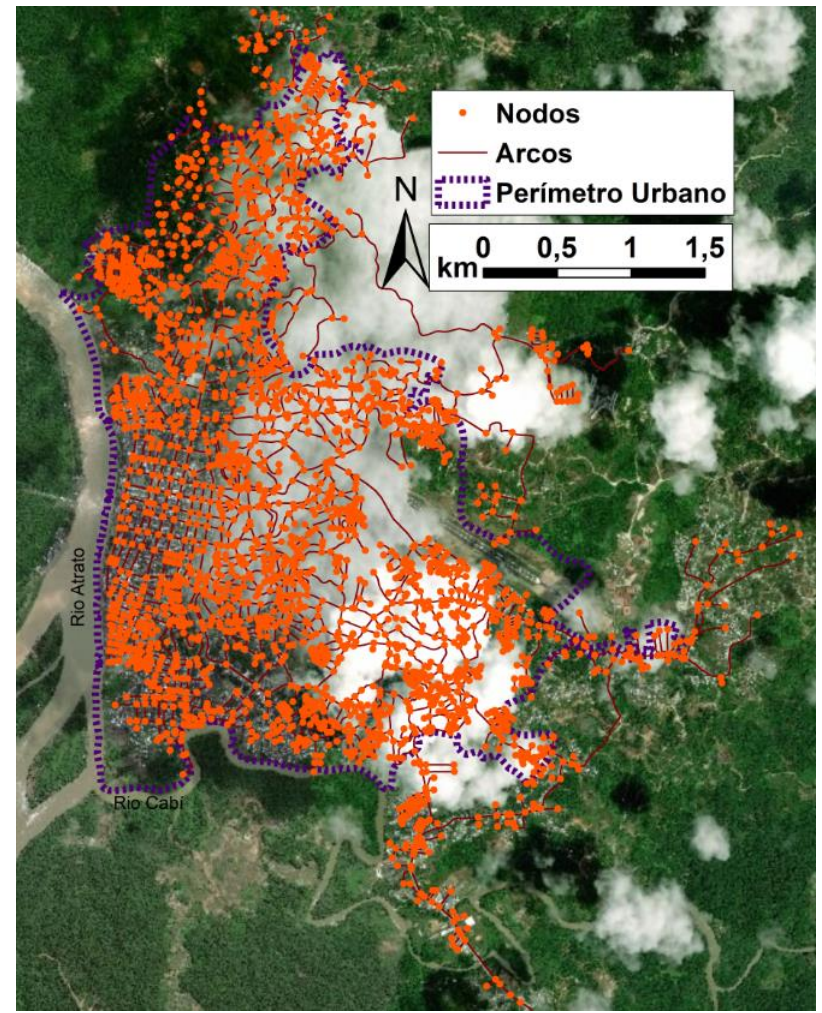

Fig. 3: Distribución espacial de la red vial (Nodos y Arcos).

\section{Construcción de curvas de accesibilidad}

La generación de las curvas de accesibilidad se centra en el cálculo del costo en tiempo medio de viaje para cada arco, el cual se determina a partir de la velocidad de operación y la longitud del arco, mediante la aplicación de la ecuación (1), donde $\mathrm{TV}_{\mathrm{i}}=$ tiempo de viaje en el arco; $\mathrm{L}_{i}=$ longitud del arco; y $V_{i}=$ velocidad operativa sobre el arco.

$$
\mathrm{Tv}_{\mathrm{i}}=\frac{\mathrm{L}_{\mathrm{i}}}{\mathrm{V}_{\mathrm{i}}} \quad \mathrm{i}=1,2,3, \ldots, \mathrm{n}
$$

Posteriormente, se construye el vector de tiempo medio de viaje, el cual determina el tiempo transcurrido sobre la red desde un nodo origen i hasta un nodo de destino j, a través del camino más corto, teniendo en cuenta la direccionalidad de las vías. Es de resaltar que dentro de la mecánica constructiva del vector, se aplica el algoritmo Dijkstra, el cual evalúa todas las posibles rutas desde el nodo origen (todos y cada uno de los nodos de la red) al nodo destino (cualquiera de los nodos de la red) y selecciona aquella con menor costo de desplazamiento (Wu y Qin, 2015) entre cada par de nodos; es decir, no se relaciona con la medición de los tiempos medios de viaje desde un nodo en específico y es por ello que se trata de una accesibilidad media global, como se explicó con anterioridad. Posteriormente, se vinculan las coordenadas geográficas de cada nodo de la red considerado en el cálculo del tiempo de viaje, obteniéndose una matriz de tamaño [ $n_{x} 3$ ], con la cual se ejecuta la herramienta Geostatical Wisard de ArcMap mediante la aplicación de los modelos geoestadísticos y se grafican las curvas isócronas de viaje. El contenido de la herramienta permite la aplicación del método de Kriging con semivariograma lineal como modelo de predicción de la variable tiempo medio de viaje en toda el área de estudio; el cálculo del semivariograma a partir de los puntos conocidos y con relación al parámetro de ponderación de semivarianza media, se calculan los valores de tiempo medio de viaje de los puntos desconocidos. La ecuación (2) es la expresión básica del método, donde $Z_{0}$ es el valor a estimar, $Z_{x}$ son los valores de los puntos conocidos, $W_{x}$ es la correlación o pesos de los puntos conocidos respecto a los puntos y $\mathrm{S}$ son los números de puntos conocidos.

$$
Z_{0}=\sum_{i=1}^{S} Z_{x} w_{x}
$$


Para el cálculo del semivariograma se aplica la ecuación (3), con la cual se caracteriza la dependencia espacial entre nodos de un mismo sistema, en donde: $Z_{(x)}$ es el valor de la variable para un nodo con coordenadas $\mathrm{x} ; \mathrm{Z}_{(\mathrm{x}+\mathrm{h})}$ es el siguiente valor de la muestra a una distancia $\mathrm{h}$; y $\mathrm{n}$ son números pares de puntos que se definen según la distancia $h$. Se considera entonces la correlación espacial entre variables definidas, generando superficies continuas a partir de nodos discretos; asumiendo que, a pesar de desconocerse el valor de la media en los puntos de interpolación, esta es constante y con variables estacionarias sin tendencias.

$$
\overline{\gamma_{(h)}}=\frac{\sum\left(\mathrm{Z}_{(\mathrm{x}+\mathrm{h})}-\mathrm{Z}_{(\mathrm{x})}\right)^{2}}{2 \mathrm{n}}
$$

\section{Cálculo del gradiente de ahorro}

El proceso constructivo de las curvas de ahorro, permiten evaluar el impacto mediante gama de colores de las intervenciones propuestas por la administración, a partir de la aplicación de la ecuación (4), en donde se relacionan los valores en tiempo de viaje obtenidos para cada escenario y en la cual: Ahorroi es el porcentaje de ahorro para el nodo $\mathrm{i}$; $\mathrm{tv}_{\mathrm{i} \text { (act) }}=$ tiempo medio de viaje en el nodo i para el escenario actual; $\mathrm{y}$ tvi(fut) es el tiempo medio de viaje en el nodo i para el escenario futuro. Posteriormente, al igual que en las curvas de accesibilidad, se ejecuta la herramienta Geostatical Wizard para construir las curvas de ahorro, aplicando nuevamente el método geoestadístico Kriging, como se mencionó con anterioridad.

$$
\operatorname{Ahorro}_{\mathrm{i}}(\%)=\left(\frac{\mathrm{tv}_{\mathrm{i}(\mathrm{act})}-\mathrm{tv}_{\mathrm{i}(\text { fut })}}{\mathrm{tv}_{\mathrm{i}(\mathrm{act})}}\right) * 100
$$

\section{Cálculo de cobertura}

Como quinta fase metodológica, se realiza el cálculo de cobertura de las variables sociodemográficas (población, área y número de viviendas). Dichas variables se encuentran cargadas a la capa shape tipo polígono como características de cada una de las entidades que conforman el shape como tal, en donde para el caso de la población la unidad es cantidad de personas, para el caso del área la unidad es $\mathrm{Km}^{2}$ y para el caso de viviendas la unidad es el número de éstas dentro de la entidad. Las variables sociodemográficas se relacionan con las curvas de accesibilidad (para ambos escenarios) y las curvas gradiente obtenidas, utilizando la herramienta Geoprocessing intersect de ArcMap; lo anterior permitió calcular el porcentaje de cobertura de dichas variables según los valores de las curvas isócronas, teniendo en cuenta los valores de población total (115.711 habitantes), número total de viviendas (26.700) y área de la zona urbana del municipio $\left(19,85 \mathrm{~km}^{2}\right)$.

\section{RESULTADOS Y DISCUSIÓN}

Teniendo en cuenta las condiciones actuales de movilidad y accesibilidad entre los sectores identificados como Admirativos y Comercio con los sectores residenciales, y con el fin de mejorar tales condiciones, mediante el Plan Integral de Movilidad del municipio, fue posible establecer la serie de intervenciones propuestas por la administración municipal para el año 2025 (Figura 4). Se plantea la construcción de 1,9 km de dobles calzadas en sectores primordiales de la ciudad que comunican de forma más expedita los espacios reproductivos con los espacios productivos (Blanco, 2016). Las intervenciones propuestas son: Doble calzada Fase I Huapango (540 metros), doble calzada Fase I Calle 30 (470 metros), doble calzada Fase I Zona Medrano (910 metros). Para la evaluación de las condiciones de accesibilidad se presentan los 2 escenarios de estudio, red base 2015 y formulación propuesta (2025).

\section{Accesibilidad, Escenario actual (año 2015)}

Como resultado de la evaluación de la accesibilidad territorial a nivel global en el escenario actual (2015), se presenta la Figura 5, en la cual se aprecia el comportamiento en tiempo medio de viaje ofertado por la red de infraestructuras del transporte del municipio a intervalos de 0,5 minutos. En color azul se resalta el área con el menor tiempo medio de viaje obtenido, la cual se localiza en el sector céntrico de la ciudad (12 minutos); como contraparte, hacia las periferias de la ciudad, se resalta en color rojo las áreas que registran el mayor tiempo medio de viaje (33 minutos), cubriendo los barrios El Futuro, Bonanza y Obapo; lo anterior, debido a la estructura misma de la red de infraestructuras del transporte, la cual posee una gran ramificación con 4 líneas de desplazamiento principales que deben soportar el tráfico del municipio desde el sector centro hacia la periferia en dirección norte, sur, oriente y viceversa. 


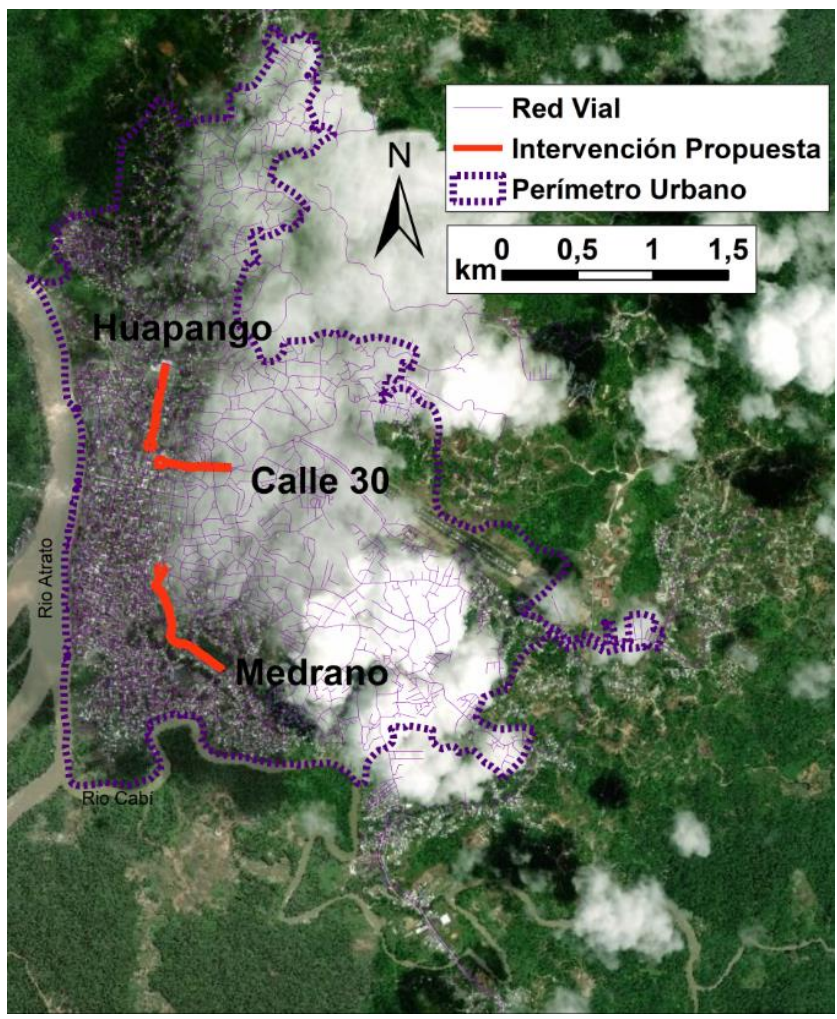

Fig. 4: Intervenciones viales propuestas por el Plan Integral de Movilidad en el corto plazo.

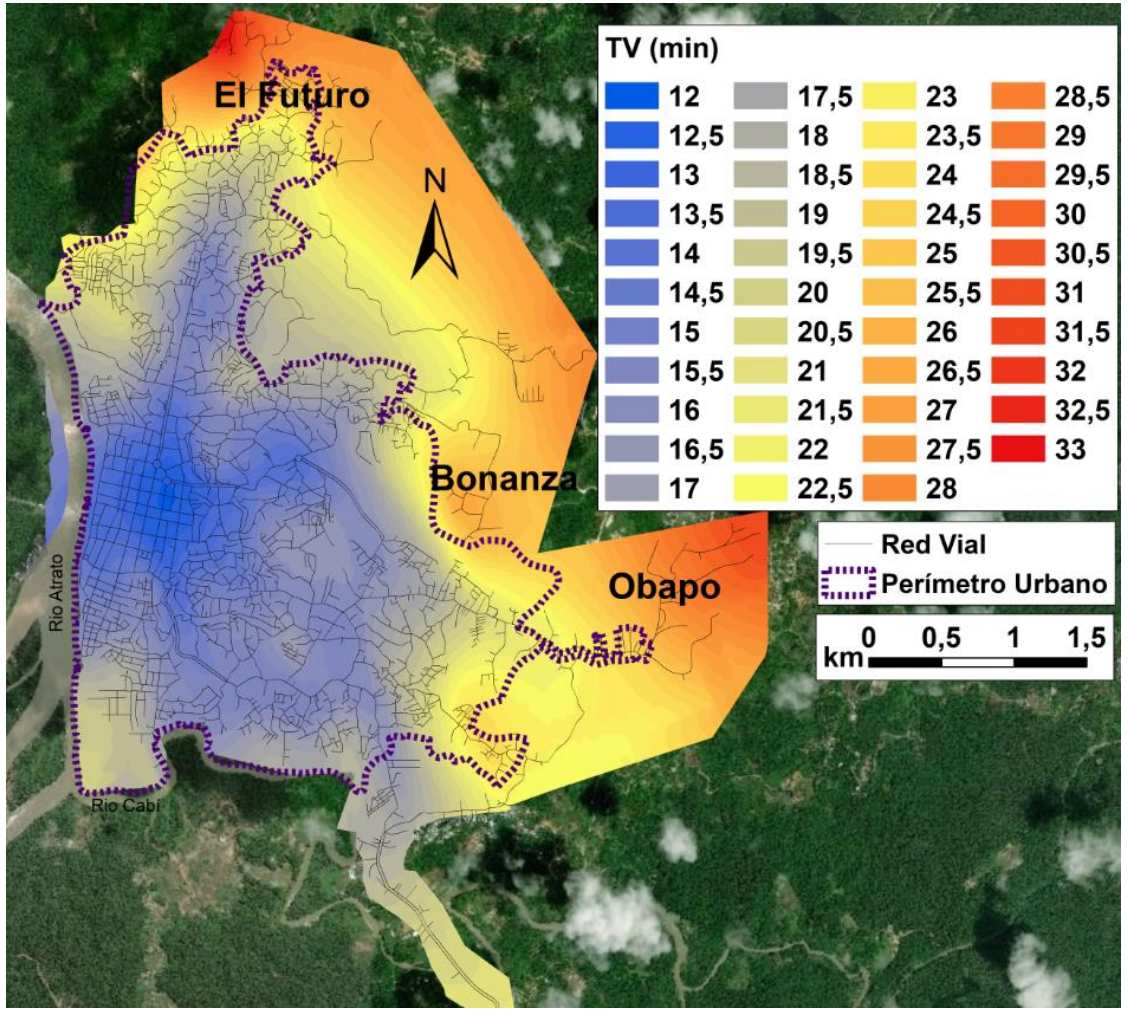

Fig.5: Curvas isócronas de accesibilidad para el escenario actual.

En la tabla 1, se presentan los resultados de cobertura de las variables sociodemográficas para el escenario actual para los diferentes rangos de tiempo medio de viaje. Se encontró que para lograr cubrir el $50 \%$ de la variable área se debían invertir más de 20 minutos de tiempo medio de viaje, valor que disminuía hasta 15 minutos si se analizaban las variables población y número de viviendas, relacionadas con el mismo porcentaje de cobertura; lo anterior indica la existencia de una menor densidad poblacional hacia los sectores periféricos de la ciudad, los cuales precisamente refieren condiciones de accesibilidad más desfavorables en el escenario actual, situación que se ve influenciada dada la estructura monocéntrica de la ciudad y las condiciones operativas actuales de la red de infraestructuras del transporte. 
Tabla 1: Porcentaje de cobertura de las variables sociodemográfica en el escenario actual (año 2015)

\begin{tabular}{|c|c|c|c|}
\hline Curva (min) & Área & Población & Viviendas \\
\hline $12-14,5$ & $7,4 \%$ & $21,3 \%$ & $22,5 \%$ \\
\hline $14,5-17$ & $27,7 \%$ & $58,1 \%$ & $60,0 \%$ \\
\hline $17-19,5$ & $41,3 \%$ & $75,6 \%$ & $76,8 \%$ \\
\hline $19,5-22$ & $56,6 \%$ & $84,7 \%$ & $86,3 \%$ \\
\hline $22-24,5$ & $73,6 \%$ & $92,0 \%$ & $93,4 \%$ \\
\hline $24,5-27$ & $91,0 \%$ & $98,8 \%$ & $98,8 \%$ \\
\hline $27-29,5$ & $99,2 \%$ & $99,4 \%$ & $99,4 \%$ \\
\hline $29,5-33$ & $100,0 \%$ & $100,0 \%$ & $100,0 \%$ \\
\hline
\end{tabular}

Accesibilidad, Escenario futuro (año 2025)

Una vez establecidas las intervenciones viales propuestas en el corto plazo por la administración municipal, se procede a realizar la evaluación de la accesibilidad en el escenario futuro (Ver Figura 6).

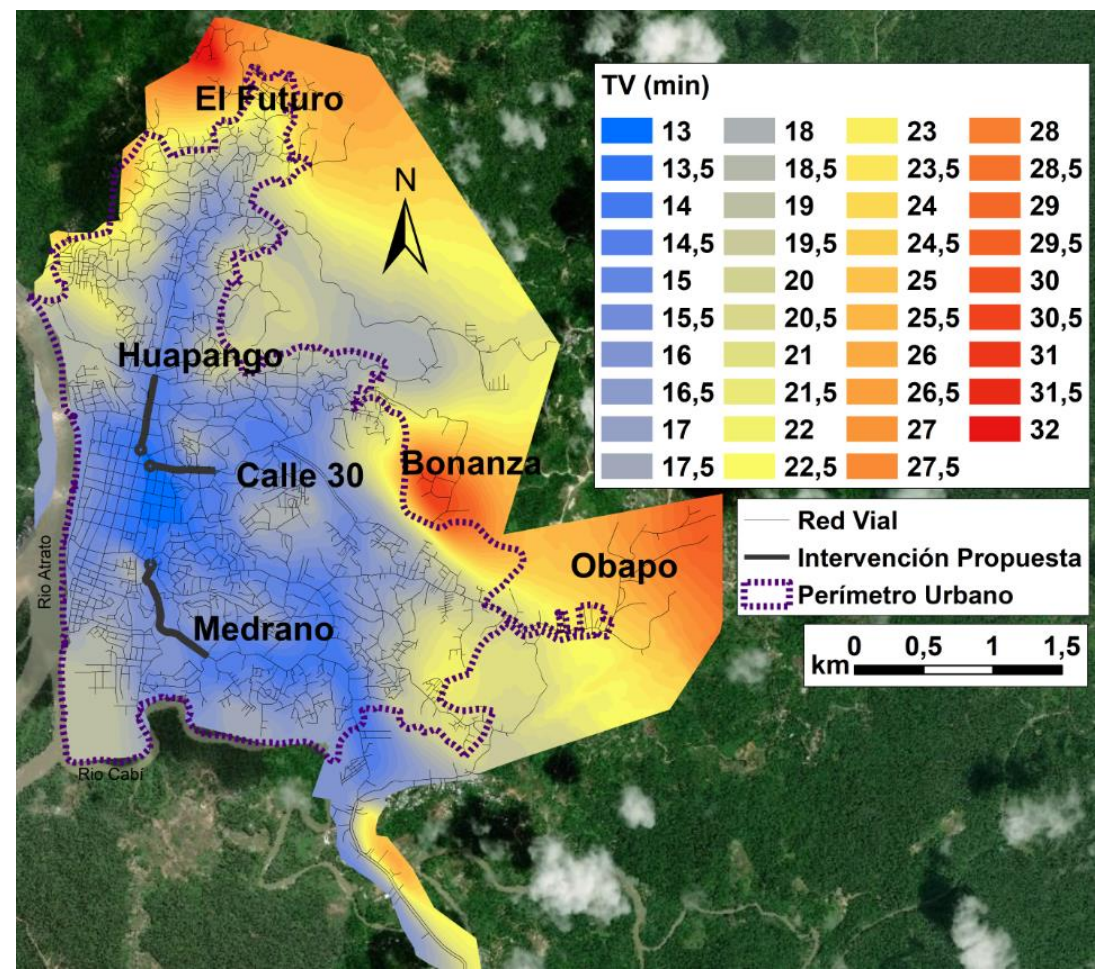

Fig.6: Curvas isócronas de accesibilidad para el escenario futuro.

Como menor valor de tiempo medio de viaje se obtuvieron 13 minutos, conservando el mismo comportamiento observado en el escenario actual, donde el sector con las condiciones de accesibilidad media global más favorables es el centro administrativo, de comercio y servicios de la ciudad; sin embargo, se aprecia un crecimiento de la zona azul hacia el sur. Por otra parte, el máximo valor de tiempo medio de viaje registra una disminución en el sector periférico de la ciudad, pasando de 33 a 32 minutos; no obstante, siguen siendo los sectores periféricos los que refieren las condiciones de accesibilidad media global más desfavorables. En la tabla 2 se presenta la relación entre los porcentajes de cobertura de las variables sociodemográficas y los rangos de tiempo medio de viaje correspondientes al escenario futuro; los porcentajes de cobertura por población y viviendas, presentan un comportamiento casi idéntico para un valor del $50 \%$ en menos de 18 minutos, es importante recordar que la población a la situación futura considera la expansión proporcionada por el Departamento Administrativo Nacional de Estadística (121.114 habitantes, 32.627 viviendas), por lo cual, a pesar de ser menor la cobertura en relación a la situación actual, se está garantizando una buena accesibilidad a un mayor número de ciudadanos. Por otra parte, el porcentaje de cobertura de la variable área, disminuye el valor del tiempo medio de viaje en relación con el escenario actual, al requerir de un tiempo de entre 18 y 20,5 minutos para alcanzar el $50 \%$ de cobertura. 
Tabla 2: Porcentaje de cobertura de las variables sociodemo-gráfica en el escenario actual (año 2025).

\begin{tabular}{|c|c|c|c|}
\hline Curva (min) & Área & Población & Viviendas \\
\hline $13,0-15,5$ & $15,7 \%$ & $21,5 \%$ & $22,1 \%$ \\
\hline $15,5-18,0$ & $37,1 \%$ & $48,8 \%$ & $50,0 \%$ \\
\hline $18,0-20,5$ & $62,0 \%$ & $82,1 \%$ & $82,5 \%$ \\
\hline $20,5-23,0$ & $79,9 \%$ & $90,4 \%$ & $91,2 \%$ \\
\hline $23,0-25,5$ & $89,9 \%$ & $96,0 \%$ & $96,2 \%$ \\
\hline $25,5-28,0$ & $97,6 \%$ & $98,2 \%$ & $98,2 \%$ \\
\hline $28,5-30,5$ & $99,8 \%$ & $99,9 \%$ & $100,0 \%$ \\
\hline $30,5-32,0$ & $100,0 \%$ & $100,0 \%$ & $100,0 \%$ \\
\hline
\end{tabular}

\section{Curvas Gradiente de Ahorro}

Con el fin de determinar el impacto generado dadas las intervenciones viales propuestas por el Plan Integrado de Movilidad de la ciudad, se calculó el gradiente de cambio (ahorro de tiempo medio de viaje) entre el escenario actual y el futuro, obteniéndose las curvas isócronas presentadas en la Figura 7.

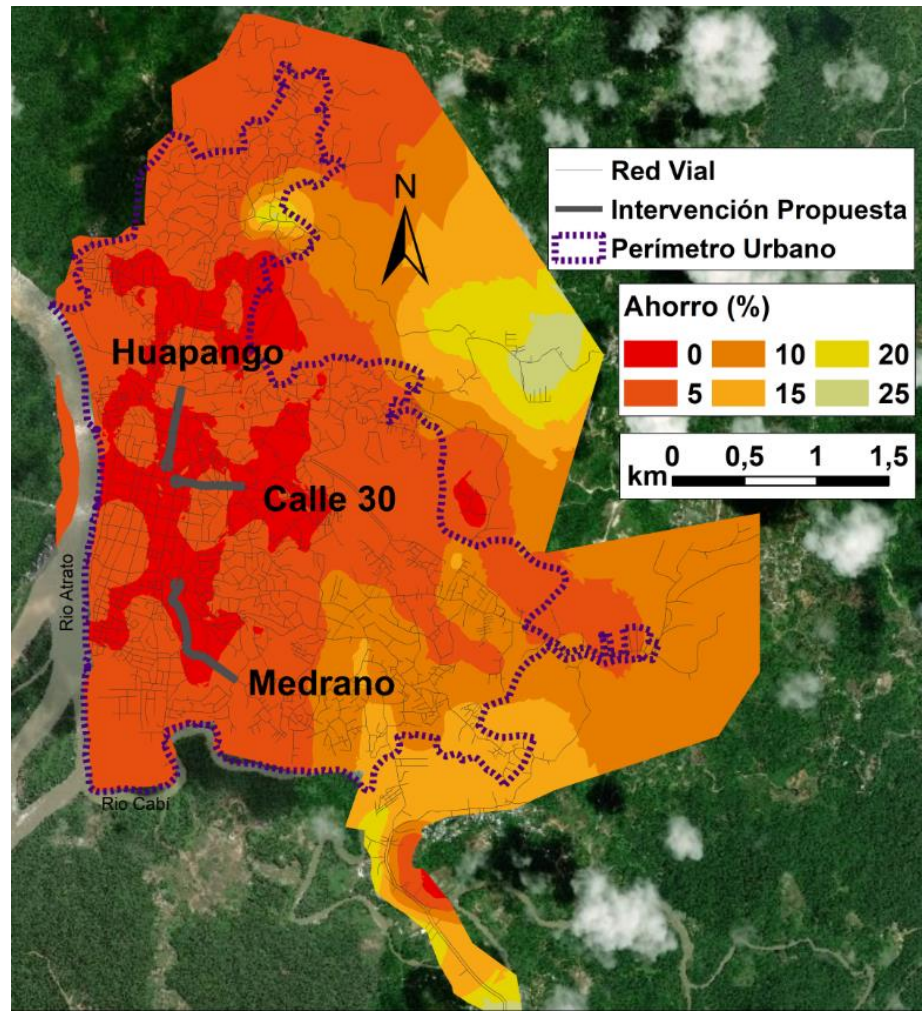

Fig.7: Curvas de ahorro porcentual de tiempo medio de viaje percibidas.

Se aprecia que la mayor parte del área urbana alcanza ahorros de entre el $5 \%$ y el $10 \%$ en relación con los valores de tiempo medio de viaje del escenario actual; se identifica que el máximo valor calculado está ubicado en el sector oriental con un valor de hasta el $25 \%$. Es posible afirmar que las intervenciones viales propuestas al horizonte 2025, generan un importante impacto sobre las condiciones de accesibilidad ofrecidas por la red de infraestructuras del transporte, aumentándose la probabilidad de que exista una movilidad más favorable y por ende una mejora en la calidad de vida al reducir los tiempos medios de viaje que los usuarios deben invertir para realizar sus actividades. Es de resaltar que este tipo de análisis necesita complementarse con la información relacionada con las zonas de expansión de la ciudad, las cuales deben estar definidas de forma oficial en el Plan de Ordenamiento Territorial del municipio, ya que una ciudad en muchas ocasiones va incorporando áreas urbanas preexistentes, generando un notable incremento demográfico de los suburbios periféricos (Kralich, 2016); para el caso de esta investigación, a pesar de no estar oficialmente definidas por la Administración Municipal, éstas áreas se identificaron hacia la periferia y según los resultados obtenidos, 
son las zonas norte y oriente las que registran mayores beneficios en relación a los valores porcentuales de gradiente en ahorro en tiempo medio de viaje.

En la tabla 3, se presentan los valores de porcentaje de cobertura de las variables sociodemográficas en relación con los porcentajes de ahorro obtenidos al comparar ambos escenarios. El comportamiento general de porcentaje de cobertura de las variables es similar, con variaciones marcadas de hasta $5 \%$ y $10 \%$ según el porcentaje de ahorro. Es de resaltar que los sectores que registran los mayores porcentajes de ahorro son sectores periféricos, es así que los barrios El Futuro y Obapo se verían beneficiados dada la construcción de las nuevas infraestructuras, mientras que el barrio Bonanza, a pesar de las nuevas infraestructuras, sigue registrando condiciones de accesibilidad desfavorables en relación con el resto del área urbana. A pesar de lo anterior, se obtuvo que más del $80 \%$ de la población y del número de viviendas registran ahorros en los tiempos medios de viaje de hasta $5 \%$ en relación con los tiempos medios de viaje del escenario actual, lo cual es considerado un valor de cobertura alto al tenerse en cuenta los valores proyectados de las variables sociodemográficas analizadas.

Tabla 3: Porcentaje de gradiente de ahorro percibido por variable sociodemográfica.

\begin{tabular}{|c|c|c|c|}
\hline Ahorro & Área & Población & Viviendas \\
\hline $5 \%$ & $89,7 \%$ & $84,3 \%$ & $82,9 \%$ \\
\hline $10 \%$ & $45,0 \%$ & $40,0 \%$ & $38,0 \%$ \\
\hline $15 \%$ & $20,8 \%$ & $26,8 \%$ & $24,2 \%$ \\
\hline $20 \%$ & $13,8 \%$ & $20,5 \%$ & $19,1 \%$ \\
\hline $25 \%$ & $9,0 \%$ & $19,7 \%$ & $17,8 \%$ \\
\hline
\end{tabular}

\section{DISCUSIÓN FINAL}

Los estudios de evaluación de impacto se han aplicado en proyectos de construcción de vías, de electrificación, provisión de agua potable, sanidad, transporte público; en donde por ejemplo, se ha buscado determinar si los proyectos han influido en aumentar la productividad de áreas específicas (Hansen et al., 2011); en algunos casos se han aplicado modelos como el Cálculo de Equilibrio General (CGE), o modelos de tratamiento y control de grupos focales aplicando el estimador Diferencia - Diferencia (DD). La teoría y tipos de estudios de evaluación del impacto generado por una infraestructura del transporte involucra el analizar si la obra es una construcción, rehabilitación o se encuentra en mantenimiento (Hansen et al., 2011); en este caso investigativo se evaluó el impacto en términos de porcentaje gradiente de cambio (tiempos medios de viaje) de obras de infraestructura que se encuentran en etapa de planeamiento. Lo anterior permite resaltar que el modelo de evaluación mediante accesibilidad geográfica, toma un enfoque de gran importancia, en donde la aplicación de técnicas geoestadísticas en instrumentos de planificación territorial, como la aquí utilizada (Modelo Kriging), expone otras posibilidades metodológicas en relación con la evaluación de impacto de diferentes alternativas de intervención sobre una red de transportes, sobre todo para soportar la propuesta o puesta en marcha de políticas públicas más acertadas.

Es válido el discutir sobre la necesidad latente de incorporar en el análisis y evaluación de impacto, los aspectos socioeconómicos de la población que se ve favorecida o no por la inserción de una obra en particular, teniendo en cuenta los usos del suelo y ubicación de los equipamientos actuales y futuros, pues la movilidad y las condiciones de accesibilidad, se encuentran directamente relacionadas con la dispersión de los usos y la articulación de los mismos con los sistemas de transportes disponibles (Blanco, 2016); sumando a esto la necesidad de comprendedor que la calidad de vida de las personas no se modifica directamente como consecuencia de construir una obra de infraestructura (Hansen et al., 2011) en particular, pero la sola construcción de las mismas hacen parte de una larga cadena de resultados en donde las condiciones geográficas juegan un importante papel en la probabilidad de mejoras las condiciones o calidad de vida de las personas. Es de reconocer entonces que analizar la ubicación de los equipamientos que permiten un análisis mínimo de inclusión social, conllevará tener más luces sobre el verdadero impacto que sobre la calidad de vida habría, siendo esto una limitante directa de la actual investigación, lo cual puede configurarse como un futuro análisis para el ajuste de los resultados obtenidos.

Las intervenciones propuestas por la administración municipal en el corto plazo, evidencian una mejora en la accesibilidad del municipio, facilitando el desplazamiento de los ciudadanos residentes en las periferias hacia el sector de productividad (centro funcional); esto, fundamentado en los resultados obtenidos en ahorro porcentual superior al $10 \%$ para estas zonas, debiéndose realizar una inversión de 42 millones USD; por otra parte, el nivel de cobertura de población y viviendas en la situación futura, presentan disminuciones mínimas en relación al escenario base, sin embargo, el incremento de la población a lo largo de los años es un elemento 
crucial de la investigación, por lo cual, las intervenciones al horizonte 2025 si garantizan unas mejores condiciones de accesibilidad de la ciudadanía, sobre todo para la comunicación desde sectores periféricos hacia el sector céntrico de la ciudad. Ahora bien, en relación con la metodología utilizada en la presente investigación y con su aplicación en otros estudios de evaluación del impacto por la construcción de infraestructuras, se encontró por ejemplo, que dada la construcción del cable aéreo en la ciudad de Manizales, en términos de accesibilidad geográfica, esta infraestructura representó un ahorro en tiempo medio de viaje de hasta el 1\% del tiempo inicial, para una inversión de 8,5 millones de USD dólares (Escobar et al., 2015). En otra investigación se aplicó la metodología para evaluar y comparar dos alternativas de infraestructuras del transporte tipo túnel, encontrándose que la alternativa más conveniente impactaría mayormente a estratos socioeconómicos medios con ahorros porcentuales de tiempo medio de viaje de hasta 12\% para una inversión proyectada de 45 millones USD (Martínez et al., 2017). En otro estudio se evaluaron cuatro diferentes alternativas de conexión viaria entre las ciudades de Manizales y Villamaría, identificando que la alternativa que refirió mayores beneficios desde el punto de accesibilidad geográfica podría llegar a reducir hasta en un 2,5\% los tiempos medios de viaje de toda la población de ambos municipios (Escobar et al., 2018). Finalmente, vale la pena resaltar que la metodología acá presentada también es útil para evaluar la ubicación de otro tipo de infraestructuras, por ejemplo, se evaluó entre cuatro puntos diferentes de posible localización de centros comerciales, cuál de ellos tendría una mejor cobertura del estrato socioeconómico objetivo, estudio que facilitó la decisión de llevar a cabo el proyecto (Montoya et al., 2017). Se evidencia que para este caso investigativo en particular, las intervenciones propuestas, se encuentran adecuadamente direccionadas, a pesar de ello es necesario tener en cuenta que los impactos observados pueden cambiar con el tiempo, es por ello que el garantizar un eficiente manejo de recursos, maximizaría el futuro impacto de las obras.

\section{CONCLUSIONES}

Con la aplicación de esta metodología se concluye sobre la posibilidad de aplicar de forma relativamente sencilla un instrumento de planificación que proporciona resultados preliminares en términos de análisis de ciudad, siendo importante el reconocer la relevancia que tiene el uso de modelos teóricos (geoestadísticos en este caso) como apoyo a la planificación, ya que proporcionan una excelente forma de validar el futuro impacto de intervenciones infraestructurales propuestas, facilitando la toma de decisiones, de igual forma, la interpretación grafica de los resultados es lo suficientemente clara para usuarios ajenos al tema. En relación con la investigación aquí presentada, se encontró que las intervenciones propuestas por la administración municipal, en términos de accesibilidad, evidencian unas mejores condiciones de la misma, lo que en un futuro facilitaría el desplazamiento de los ciudadanos residentes en los sectores periféricos de la ciudad con el centro funcional, registrando porcentajes de ahorro superiores al $10 \%$ en relación con los tiempos medios de viaje del escenario actual; en donde, a pesar que los niveles de cobertura de las variables población y vivienda, registran cambios mínimos en relación al escenario actual, el incremento esperado de la población al año 2025, hace que el porcentaje cubierto de estas variables si aumente. Se pone de manifiesto que la investigación presentada es un paso inicial importante en la evaluación del impacto que las obras propuestas podrían imprimir a la ciudad, lo cual debe ser complementado con el conocimiento detallado de las necesidades o requerimiento de movilidad de la población, y articulado con la accesibilidad calculada, ya que ésta muestra la capacidad actual y futura de la red de infraestructuras para, por lo menos en términos de tiempo medio de viaje, satisfacer dichas necesidades de desplazamiento.

\section{AGRADECIMIENTOS}

Los autores expresan sus agradecimientos a los jóvenes pertenecientes a los grupos de investigación en Planificación Urbana y Movilidad Sostenible adscritos a la Universidad Nacional de Colombia, Sede Manizales.

\section{REFERENCIAS}

Alcaldía de Quibdó. Plan Integral de Movilidad del Municipio de Quibdó - Choco. Parte I- Diagnostico. Alcaldía de Quibdó, Universidad Nacional de Colombia - Sede Manizales, (2015)

Arcidiacono, C. y Porto S. M., A model to manage crop-shelter spatial development by multi-temporal coverage analysis and spatial indicators, doi:10.1016/j.biosystemseng.2010.07.007, Biosystems Engineering, 107(2), 107-122 (2010)

Batty, M., Accessibility: in search of a unified theory, doi:10.1068/b3602ed, Environment and Planning B. Planning and Design, 36(2), 191-194 (2009)

Blanco, J., Urbanización y movilidad: contradicciones bajo el modelo automóvil - intensivo. ISSN: 1852-7175, Revista Transporte y Territorio, 15, 96-113 (2016)

Brida, J. G., Deidda M. y Pulina M., Tourism and transport systems in mountain environments: Analysis of the economic efficiency of cableways in South Tyrol, doi:10.1016/j.jtrangeo.2014.02.004, Journal of Transport Geography, 36, 1-11 (2014) 
Escobar, D.A., Tapasco O. y Giraldo J., Medición de Desempeño del Sistema de Transporte Cable Aéreo de la Ciudad de Manizales en Colombia, usando Tres Enfoques: Analítico, Simulado y de Accesibilidad Urbana, doi:10.4067/S071807642015000600020, Información Tecnológica, 26(6), 199-210 (2015)

Escobar, D.A., Cardona S. y Moncada C.A., Evaluation of Road Infrastructure Alternatives Through a Saving Gradient in Travel Times. Case Study: Second Connection Villamaría-Manizales, Colombia, doi:10.5539/mas.v12n8p103, Modern Applied Science, 12(8), 103-115 (2018)

Dupuy, G., El urbanismo de las redes: teorías y métodos, 1a edición, Oikos-Tau Editores, Barcelona, España (1998)

Geetam, T. y Deepty J., Accessibility and safety indicators for all road users: case study Delhi BRT, doi:10.1016/j.jtrangeo.2011.11.020, Journal of Transport Geography, 22, 87 - 95 (2012)

Geurs, K. T. y Van Wee B., Accessibility evaluation of land-use and transport strategies: review and research directions, doi:10.1016/j.jtrangeo.2003.10.005, Journal of Transport Geography. 12(2), 127-140. (2004)

Gutiérrez, A. ¿Qué es la movilidad? Elementos para (re) construir las definiciones básicas del campo del transporte. ISSN: 2027-145X, Bitácora Urbano-Territorial, 21(2), 61-74 (2012)

Handy, S. y Niemeier D., Measuring accessibility: an exploration of issues and alternatives, doi:10.1068/a291175, Environment and planning A, 29(7), 1175-1194, (1997)

Hansen, H., Winckler A. y White $\mathrm{H}$., Impact evaluation of infrastructure interventions, https://doi.org/10.1080/19439342.2011.547659, Journal of Development Effectiveness, 3(1), 1 - 8 (2011)

Hansen, W., How accessibility shapes land use, doi:10.1080/01944365908978307, Journal of the American Institute of Planners, 25(2), 73-76 (1959)

Hansz, M., Hernández D. y Rubinstein E., ¿Qué implica la accesibilidad en el diseño e implementación de políticas públicas urbanas?, doi:10.18235/0001469, Banco Interamericano de Desarrollo, Nota técnica IDB-TN-1562, 1-46 (2018)

Hartmann, A. y Yng Ling F.Y., Value creation of road infrastructure networks: A structural equation approach, doi:10.1016/j.jtte.2015.09.003, Journal of traffic and transportation engineering (English Edition), 3(1), 28-36, (2016)

Kralich, P. Urbanización y transporte. Algunos aportes conceptuales. ISSN: 1852-7175, Revista Transporte y Territorio, 15, 41-67 (2016)

Kwok, R.C. y Yeh A.G., The use of modal accessibility gap as an indicator for sustainable transport development, doi:10.1068/a3673, Environment and Planning A, 36(5), 921-936 (2004)

Lucas, K. Transport and social exclusion: Where are we now?, doi:10.1016/j.tranpol.2012.01.013, Transport Policy, 20, 105-113 (2012)

Lucas, K., Van Wee B. y Maat K.A., A method to evaluate equitable accessibility: combining ethical theories and accessibility-based approaches, doi:10.1007/s11116-015-9585-2, Transportation, 43(3) (2016)

Martínez, S., Escobar D.A. y Tamayo J.A., Evaluación Comparativa de dos Alternativas de Infraestructura Tipo Túnel a partir de un Análisis de Accesibilidad Urbana, doi:10.4067/S0718-07642017000400018, Información tecnológica, 28 (4), 157-168 (2017)

Montoya, J., Escobar D.A. y Moncada C.A., Proposed location of new shopping centers, application of an urban territorial accessibility analysis. ISSN: 0798-1015, Revista Espacios, 38(51), 4 (2017)

Moreno, S.L. y Jácome J.M., Usos de información georreferenciada para prestación de servicios a la población: Una revisión de literatura, doi:10.21138/GF.572, GeoFocus - Revista Internacional de Ciencia y Tecnología de la Información Geográfica, 20, 201-229 (2017)

Murray, T. y Wu X., Accessibility tradeoffs in public transit planning, doi:10.1007/s101090300105, Journal of Geographical Systems. 5, 93 - 107 (2003)

Pirie, G.H., Measuring accessibility: a review and proposal. doi:10.1068/a110299, Environment and Planning A, 11(3), 299-312 (1979)

Schürmann, C., Spiekermann K. y Wegener M., Accessibility indicator. SASI Deliverable D5. Report to the European Commission, Berichte aus dem Institut für Raumplanung, 39 (1997)

Van Wee, B., Accessible accessibility research challenges, doi:10.1016/j.jtrangeo.2015.10.018, Journal of Transport Geography, 51, 9-16 (2016)

Wu, Q., Qin G. y Li H., An Improved Dijkstra's algorithm application to multi-core processors. Metallurgical \& Mining Industry, 9, 76-81 (2015)

Yigitcanlar, T., Sipe N., Evans R. y Matt P., A GIS-based land use and public transport accessibility indexing model, doi:10.1080/07293682.2007.9982586, Australian Planner, 44(3), 30-37 (2010)

Walsh, S., Cullinan J. y Flannery D., The Impact of Proposed Higher Education Reforms on Geographic Accessibility to Universities in Ireland, doi:10.1007/s12061-016-9193-3, Applied Spatial Analysis and Policy, 10(4), 515-536 (2017) 